PROCEEDINGS OF THE

AMERICAN MATHEMATICAL SOCIETY

Volume 126, Number 4, April 1998, Pages 989-997

S 0002-9939(98)04180-X

\title{
ON THE JACOBIAN MODULE ASSOCIATED TO A GRAPH
}

\author{
ARON SIMIS
}

(Communicated by Wolmer V. Vasconcelos)

\begin{abstract}
We consider the jacobian module of a set $\mathbf{f}:=\left\{f_{1}, \ldots, f_{m}\right\} \in$ $R:=k\left[X_{1}, \ldots, X_{n}\right]$ of squarefree monomials of degree 2 corresponding to the edges of a connected bipartite graph $G$. We show that for such a graph $G$ the number of its primitive cycles (i.e., cycles whose chords are not edges of $G)$ is the second Betti number in a minimal resolution of the corresponding jacobian module. A byproduct is a graph theoretic criterion for the subalgebra $k[G]:=k[\mathbf{f}]$ to be a complete intersection.
\end{abstract}

\section{INTRODUCTION}

Let $\mathbf{f}=\left\{f_{1}, \ldots, f_{m}\right\} \subset R=k[\mathbf{X}]=k\left[X_{1}, \ldots, X_{n}\right]$ be a set of polynomials, where $k$ is a field. The jacobian module $\mathfrak{J}(\mathbf{f})$ of $\mathbf{f}$ is the cokernel of the map $R^{m} \rightarrow R^{n}$ defined by the transposed jacobian matrix ${ }^{t} \Theta(\mathbf{f})={ }^{t} \Theta\left(f_{1}, \ldots, f_{m}\right)$ of the polynomials $\mathbf{f}$.

If $\mathbf{f}$ is a set of linearly independent homogeneous polynomials of the same degree, then $\mathfrak{J}(\mathbf{f})$ does not depend on the choice of a minimal set of homogeneous generators. The underlying theme of this work is the understanding of how combinatorial properties of an ideal (or $k$-subalgebra) of $R$ minimally generated by quadrics $\mathbf{f}=\left\{f_{1}, \ldots, f_{m}\right\}$ translate to the numerical invariants of the minimal homogeneous free resolution of $\mathfrak{J}(\mathbf{f})$.

One finds that these considerations have a degree of success in the case where $\mathbf{f}=\left\{f_{1}, \ldots, f_{m}\right\}$ correspond to the edges of a simple connected graph $G$, generating the so-called edge-ideal $I(G)$ (cf. [3], [4]). In this setup, the simpler notation $\mathfrak{J}(G):=\mathfrak{J}(\mathbf{f}),{ }^{t} \Theta(G):={ }^{t} \Theta(\mathbf{f})$ will be used. It is shown that if $G$ is a connected bipartite graph then the second module of syzygies of $\mathfrak{J}(G)$ is generated by the socalled polar syzygies (to be defined below). As a consequence, one also obtains that the second Betti number in the minimal free resolution of $\mathfrak{J}(G)$ over the polynomial ring $R$ coincides with the number of primitive (i.e., chordless) cycles of $G$, and that $\mathfrak{J}(G)$ has homological dimension at most 2 if and only if the number of primitive cycles of $G$ coincides with the usual rank of $G$ in the sense of graph theory. In particular, one obtains a way of translating the computation of the number of primitive cycles of a connected bipartite graph into the computation of the number of minimal generating relations of a matrix whose entries are indeterminates. Moreover,

Received by the editors June 1, 1996 and, in revised form, September 27, 1996.

1991 Mathematics Subject Classification. Primary 13H10; Secondary 13D40, 13D45, 13H15.

The author was partially supported by CNPq, Brazil. 
from each such generating relation one can read off the order of the corresponding primitive cycle.

A byproduct is a criterion for the subalgebra $k[G]$ to be a complete intersection. This relates to [2], where the authors consider the case of bipartite planar graphs by entirely different methods.

An earlier version of this work underwent important criticism by someone who wishes to remain anonymous; the present version reflects partially this criticism, for which I am indebted.

\section{Preliminaries}

We collect a few facts concerning the jacobian matrix of a set of monomials for which a suitable reference in the literature has not been found. Let $\mathbf{X}=X_{1}, \ldots, X_{n}$ be indeterminants over a field $k$. By a monomial in $k[\mathbf{X}]$ we mean a power product $\mathbf{X}^{\mathbf{a}}=X_{1}^{a_{1}} \cdots X_{n}^{a_{n}}$, with $a_{i} \in \mathbb{N}$.

The following result is quite particular about the jacobian matrix of a set of monomials.

Lemma 1.1. Let $\mathbf{f}=\left\{f_{1}, \ldots, f_{m}\right\}$ be a set of monomials in $k[\mathbf{X}]$. Then every minor of the jacobian matrix of $\mathbf{f}$ is of the form $\alpha \mathbf{X}^{\mathbf{a}}$, where $\alpha$ belongs to the prime ring of $k$.

Proof. One endows the ring $k[\mathbf{X}]$ with the $\mathbb{N}^{n}$-gradation in which $k[\mathbf{X}]_{\mathbf{a}}=k \mathbf{X}^{\mathbf{a}}$. A homogeneous element in this gradation will be called multihomogeneous. Now, we claim that the jacobian matrix is also multihomogeneous, i.e., that any $2 \times 2$ minor is a multihomogeneous element of $k[\mathbf{X}]$. This will imply that any minor is multihomogeneous, hence must be of the form $\alpha \mathbf{X}^{\mathbf{a}}$, for some $\mathbf{a} \in \mathbb{N}^{n}$ and some $\alpha \in k$. To conclude, the definition of derivatives easily implies that $\alpha$ actually belongs to the prime ring of $k$. Thus, let, say, the $2 \times 2$ minor be that of columns 1,2 and rows 1,2 . Letting $f_{1}=\mathbf{X}^{\mathbf{a}}$ and $f_{2}=\mathbf{X}^{\mathbf{b}}$, the minor is a binomial with terms of multidegrees $\left(a_{1}-1+b_{1}, a_{2}+b_{2}-1, \ldots\right)$ and $\left(a_{1}+b_{1}-1, a_{2}-1+b_{2}, \ldots\right)$, respectively. Since the latter are equal, the minor is multihomogeneous.

Consider the map $\mathcal{L}$ that associates to a monomial $\mathbf{X}^{\mathbf{a}}$ its exponent vector $\mathbf{a} \in$ $\mathbb{N}^{n}$. Given a finite set $\mathbf{f}=\mathbf{X}^{\mathbf{a}_{1}}, \ldots, \mathbf{X}^{\mathbf{a}_{m}}$ of monomials, one can look at the integer matrix $\mathcal{L}(\mathbf{f})=\left(\mathbf{a}_{1}, \ldots, \mathbf{a}_{m}\right)$. One may call $\mathcal{L}(\mathbf{f})$ the log-matrix of $\mathbf{f}$. Note that if $G$ is a simple graph then the log-matrix of the generators of $k[G]$ is precisely the incidence matrix of $G$.

A consequence is as follows.

Proposition 1.2 (char $k=0)$. Let $\mathbf{f}$ be a set of monomials. Then the jacobian matrix of $\mathbf{f}$ and the log-matrix of $\mathbf{f}$ have the same rank.

Proof. Identify $\mathbb{Z}$ with a subring of $k$. Then ${ }^{t} \Theta(\mathbf{f})$ has entries in $\mathbb{Z}$ and the canonical homomorphism $\mathbb{Z}[\mathbf{X}] \rightarrow \mathbb{Z}$ implies that $\operatorname{rank}(\mathcal{L}(\mathbf{f})) \leq \operatorname{rank}\left({ }^{t} \Theta(\mathbf{f})\right)$. Conversely, let $r=\operatorname{rank}^{t} \Theta(\mathbf{f})$ and let $\Delta$ be a nonzero $r \times r$ minor of ${ }^{t} \Theta(\mathbf{f})$. Then $\Delta$ is of the form $\alpha \mathbf{X}^{\mathbf{a}}$, with $\alpha \in \mathbb{Z} \backslash\{0\}$ by Lemma 1.1. Therefore, $\epsilon(\Delta)=\alpha \neq 0$. But $\epsilon(\Delta)$ is an $r \times r$ minor of $\mathcal{L}(\mathbf{f})$.

Note that the proposition fails in positive characteristic on taking

$$
\mathbf{f}=\left\{X_{1} X_{2}, X_{1} X_{3}, X_{2} X_{3}\right\}
$$


The previous results allow for the following alternative characterization of a connected bipartite graph.

Proposition 1.3 (char $k=0)$. Let $G$ be a connected graph. The following conditions are equivalent:

(i) $G$ is bipartite.

(ii) $\mathfrak{J}(G)$ has rank one.

(iii) $\mathfrak{J}(G)$ has positive rank.

Proof. It is well-known that, for a connected graph $G$ on $n$ vertices, its incidence matrix has rank at least $n-1$, and that it has exactly rank $n-1$ if and only if $G$ is bipartite. Therefore, the result is a straightforward consequence of Proposition 1.2.

\section{The SYZYGIES OF THE JACOBIAN MODUlE}

The following result gives a graph-theoretic characterization of a connected graph whose jacobian module has the smallest possible homological dimension.

Proposition 2.1 (char $k=0$ ). Let $G$ be a connected graph. The following conditions are equivalent:

(i) $\mathfrak{J}(G)$ has homological dimension one.

(ii) $G$ has at most one cycle, and this cycle is odd.

Proof. (i) $\Rightarrow$ (ii). Let $n$ be the number of vertices of $G$. Since $G$ is connected, the number $m$ of edges is at least $n-1$. If this number is $n-1$ then $G$ is a tree. So, assume that $m \geq n$. The homological dimension being 1 , we must have $m=n$. But in this case it is well-known or clear that $G$ admits at most one cycle. By Proposition 1.3, this cycle must be odd.

(ii) $\Rightarrow$ (i). The case of a tree $G$ is easy, directly. Indeed, induction on the number of vertices, passing to a subtree $G \backslash\left\{X_{i}\right\}$ with $X_{i}$ a vertex of degree one, shows that in fact any individual $(n-1) \times(n-1)$ minor of ${ }^{t} \Theta(G)$ is nonzero. Thus, assume that $G$ contains a unique cycle and that cycle is odd. In this case the jacobian matrix of $I(G)$ is a square matrix. By Proposition 1.3, the determinant of ${ }^{t} \Theta(G)$ is nonzero; hence $\mathfrak{J}(G)$ has homological dimension one.

Remark 2.2. By [4], condition (ii) above characterizes a connected graph $G$ for which the edge-ideal $I(G)$ is of linear type, i.e., for which the symmetric algebra of $I(G)$ is a torsion-free $R$-algebra. Thus, Proposition 2.1 shows that this condition is also equivalent to saying that the jacobian module $\mathfrak{J}(G)$ has homological dimension one.

2.1. Polar syzygies. Now, quite generally for the sake of definition, let $\mathbf{f}=$ $\left\{f_{1}, \ldots, f_{m}\right\}$ be a set of polynomials in $R=k[\mathbf{X}]$ such that $f_{j}(\mathbf{0})=0, \forall j$. Let $\mathcal{D}(I):=\operatorname{Im}\left({ }^{t} \Theta(\mathbf{f})\right) \subset \sum_{i=1}^{n} R d X_{i}$ stand for the submodule generated by the differentials of $\mathbf{f}$. One is interested in finding a structured set of generators of its first syzygy module $\mathcal{Z}:=Z_{1}(\mathcal{D}(I)) \subset R^{m}=\sum_{j} R T_{j}$.

For that purpose, one considers the $k$-linear map $\lambda: k[\mathbf{T}]=S\left(R^{m}\right) \rightarrow R^{m}$ given by $\lambda(F):=\sum_{j} \frac{\partial F}{\partial T_{j}}\left(f_{1}, \ldots, f_{m}\right) T_{j} \in R^{m}$. Moreover, let $J \subset k[\mathbf{T}]$ denote the ideal of polynomial relations of $\mathbf{f}$. By the usual rules of composite derivatives, if $F \in J$ then $\sum_{j=1}^{m} \frac{\partial F}{\partial T_{j}}\left(f_{1}, \ldots, f_{m}\right) d f_{j}=0$. This means that restriction induces a map $J \rightarrow \mathcal{Z}$. Any element in the image of $J$ will be called a polar syzygy. 
Finally, we claim that the latter map still induces a quotient map

$$
\bar{\lambda}: J /(\mathbf{T}) J \rightarrow \mathcal{Z} /(\mathbf{X}) \mathcal{Z} .
$$

To see this, let $F \in(\mathbf{T}) J$, say, $F=\sum_{k} G_{k} F_{k}$, with $G_{k} \in(\mathbf{T})$ and $F_{k} \in J$ for every $k$. Then, by the derivative rules and the vanishing $F_{k}(\mathbf{f})=0$,

$$
\begin{aligned}
\lambda(F) & =\sum_{j} \frac{\partial F}{\partial T_{j}}(\mathbf{f}) T_{j}=\sum_{k}\left(\sum_{j} \frac{\partial F_{k}}{\partial T_{j}}(\mathbf{f}) T_{j}\right) G_{k}(\mathbf{f}) \\
& =\sum_{k} G_{k}(\mathbf{f}) \lambda\left(F_{k}\right) \in(\mathbf{X}) \lambda(J) \subset(\mathbf{X}) \mathcal{Z} .
\end{aligned}
$$

The map (1) will be called the polar map (associated to $\mathbf{f}$ ). If $\mathbf{f}$ are homogeneous polynomials of the same degree, one says that $\mathfrak{J}(\mathbf{f})$ is polarizable if the polar map is an isomorphism. When $\mathbf{f}$ are homogeneous of the same degree, $J$ (resp. $\mathcal{Z}$ ) is a homogeneous ideal of $k[\mathbf{T}]$ (resp. a graded submodule of $\sum_{j} R T_{j}$ ). When needed, one denotes by $J_{q}$ and $\mathcal{Z}_{q}$ the respective $q$ th graded pieces.

The central question in this regard asks when the polar map is an isomorphism. That it may fail to be so in general is shown by the edge-ideal of the non-bipartite graph below:

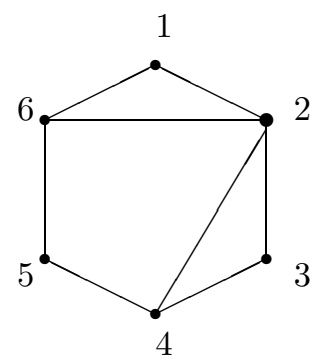

An easy calculation shows here that $\mu(J)=3$ and $\mu(\mathcal{Z})=2$; hence the polar map fails to be an isomorphism. Here, the "extra" minimal generator of $J$ coming from the even walk (not a cycle!) with vertices $X_{1}, X_{2}, X_{3}, X_{4}, X_{2}, X_{6}$ maps to a deep combination of the 2 minimal generators of the module $\mathcal{Z}$. Of these, one is of degree 3 , hence cannot be a polar syzygy. Thus, the polar map is neither injective nor surjective.

In view of this example, the following result seems to be of interest.

Theorem 2.3. If $G$ is a connected bipartite graph, then $\mathfrak{J}(G)$ is polarizable.

In order to prove this result, one first needs a good grip on a set of generators of the ideal $J \subset k[\mathbf{T}]$ of relations. For bipartite graphs, the peculiar form of the generators of $J$ as obtained in [5] allows for a substantial reduction of the number of generators in terms of the so-called primitive cycles (a cycle $C$ of a graph $G$ is a primitive cycle of $G$ if no chord of $C$ is an edge of $G$ ). Recall that the order of a cycle $C$ of $G$ is the number of edges of $G$ belonging to $C$.

Here is the precise statement.

Lemma 2.4. Let $G$ be a connected bipartite graph. Then the residue classes of the (binomial) relations corresponding to the primitive cycles of $G$ of order $2 q$ form a vector basis of $(J /(\mathbf{T}) J)_{q}=J_{q} / \sum_{j} T_{j} J_{q-j}$. In particular, $J$ is minimally generated by the relations corresponding to the primitive cycles. 
Proof. By [5, Proposition 3.1] and since $G$ is bipartite, one knows that $J$ is generated by the binomial relations coming from the cycles of $G$. Now, if $C_{2 q}$ is not a primitive cycle, then it is subdivided into two other cycles $C_{2 q^{\prime}}^{\prime}, C_{2 q^{\prime \prime}}^{\prime \prime}$ (necessarily even) with $2 q=2 q^{\prime}+2 q^{\prime \prime}-2$, the two sharing a common edge which is a chord of $C_{2 q}$. In this situation, one can see that the relation coming from $C_{2 q}$ is in the ideal generated by those coming from the two smaller cycles. This shows that the relations coming from the primitive cycles generate the ideal $J$; hence the (images of) these relations corresponding to the-primitive cycles of order $2 q$ span the vector space $J_{q} / \sum_{j} T_{j} J_{q-j}$. It remains to see that they are linearly independent. Now, an even primitive cycle is determined by a complete set of its alternate edges, i.e., two primitive cycles in a bipartite graph sharing a common 1-factor are equal. Therefore, for any two primitive cycles of order $2 q$, the corresponding binomial relations are such that their constituent monomials are all pairwise distinct (i.e., have different supports). Thus, a $k$-linear combination of such distinct binomials is also one of distinct monomials. Now, by induction on $q$, one may assume that the primitive cycles of any order $2 p<2 q$ already form a $k$-basis of $J_{p} / \sum_{j} T_{j} J_{p-j}$. Let it be given that

$$
\sum_{l=1}^{s} \nu_{l} F_{l}=\sum_{k_{1}} G_{k_{1}} F_{k_{1}}+\ldots+\sum_{k_{q-2}} G_{k_{q-2}} F_{k_{q-2}},
$$

where $F_{l}$ (resp. $F_{k_{u}}$ ) is the relation yielded by a primitive cycle of order $2 q$ (resp. $2(u+1))$ and $\nu_{l} \in k$. Moreover, one may assume that the left-hand side has the smallest number $s$ of nonzero coefficients $\nu_{l}$ for which a relation (2) holds. In particular, $\nu_{l} \neq 0$ for every $l$. Let $F_{1}=M_{1}-N_{1}$, with $M_{1}, N_{1}$ monomials such that $\operatorname{gcd}\left(M_{1}, N_{1}\right)=1$. Then there exist $F_{k_{u}}=M_{k_{u}}-N_{k_{u}}, F_{k_{v}}=M_{k_{v}}-N_{k_{v}}$ such that $M_{1}=L_{k_{u}} M_{k_{u}}, N_{1}=-L_{k_{v}} N_{k_{v}}$, for suitable monomials $L_{k_{u}}, L_{k_{v}}$. Clearly, $L_{k_{u}} F_{k_{u}}+L_{k_{v}} F_{k_{v}} \in J_{q}$, and since

$$
L_{k_{u}} F_{k_{u}}+L_{k_{v}} F_{k_{v}}=F_{1}-\left(L_{k_{u}} N_{k_{u}}-L_{k_{v}} M_{k_{v}}\right) \text {, }
$$

one has $L_{k_{u}} N_{k_{u}}-L_{k_{v}} M_{k_{v}} \in J_{q}$. If this binomial actually belongs to $\sum_{j} T_{j} J_{q-1}$, then by cancelling $\nu_{1} F_{1}$ on both sides of (2), one would get a relation of the same form as (2) only with the left-hand side having fewer terms. Therefore, it must be the case that $F:=L_{k_{u}} N_{k_{u}}-L_{k_{v}} M_{k_{v}} \in J_{q} \backslash \sum_{j} T_{j} J_{q-1}$. But then (3) gives a relation such as (2) whose left-hand side is $\nu_{1} F_{1}+\nu F$, for suitable $\nu \in k$. So, the number $s$ of terms in the left-hand side of (2) is at most 2, and one may assume the relation is of the form

$$
\nu_{1} F_{1}+\nu_{2} F_{2}=L_{k_{u}} F_{k_{u}}+L_{k_{v}} F_{k_{v}}
$$

with $F_{k_{u}}$ and $F_{k_{v}}$ corresponding as before to smaller cycles. Since no further cancelling is possible in the right-hand side alone as the monomials in the left-hand side are all distinct, it follows that the smaller cycles could only be produced by chords of the cycles corresponding to the binomials $F_{1}, F_{2}$. This is a contradiction, as they are primitive cycles by hypothesis.

Proof of the theorem. Since we are dealing with a bipartite graph, the two sets of variables giving the appropriate bipartition will be object of emphasis. Thus, $\mathbf{X}$ will be replaced by $\mathbf{X}, \mathbf{Y}$. The transposed jacobian matrix of $I(G)$ can then be 
written in the following form:

$\left(\begin{array}{cccc|cccc|c|cccc}Y_{u_{1}} & Y_{u_{2}} & \ldots & Y_{u_{d_{1}}} & 0 & 0 & \ldots & 0 & \ldots & 0 & 0 & \ldots & 0 \\ 0 & 0 & \ldots & 0 & Y_{v_{1}} & Y_{v_{2}} & \ldots & Y_{v_{d_{2}}} & \ldots & 0 & 0 & \ldots & 0 \\ 0 & 0 & \ldots & 0 & 0 & 0 & \ldots & 0 & \ldots & 0 & 0 & \ldots & 0 \\ 0 & 0 & \ldots & 0 & 0 & 0 & \ldots & 0 & \ldots & Y_{w_{1}} & Y_{w_{2}} & \ldots & Y_{w_{d_{n}}} \\ & & & & & & & & & & & & \\ & \mathbf{X}_{1} & & & & \mathbf{X}_{2} & & & \ldots & & \mathbf{X}_{n} & & \\ & & & & & & & & & & & & \end{array}\right)$,

where $d_{i}$ is the degree of the vertex $X_{i}$ and $\mathbf{X}_{i}$ denotes a matrix with only $X_{i}$ or 0 as entries. The polynomial ring $R=k[\mathbf{X}, \mathbf{Y}]$ is $\mathbb{N}^{r} \times \mathbb{N}^{s}$-graded, by setting $\operatorname{bideg}(f):=\left(\operatorname{deg}_{\mathbf{X}}(f), \operatorname{deg}_{\mathbf{Y}}(f)\right)$ (here deg stands for multidegree). The $\partial / \partial X_{i}$-row (resp. $\partial / \partial Y_{j}$-row ) will be called the $X_{i}$-row (resp. $Y_{j}$-row). Then $\mathcal{Z}$ admits a set of generating syzygies whose coordinates are monomials and have constant bidegree in $\mathbf{X}, \mathbf{Y}$ (see the proof of Lemma 1.1).

One first shows that the polar map (1) is surjective. The argument will use induction on the number of edges of the graph, noting there is nothing to be proved if $G$ has less than 4 edges, since then it must be a tree (cf. Proposition 2.1 and Remark 2.2). Thus, assume that $G$ has at least 4 edges. Let $\mathbf{z}=\left(g_{1, u_{1}}, \ldots, g_{1, u_{d_{1}}} ; \ldots ; g_{n, w_{1}}, \ldots, g_{n, w_{d_{n}}}\right) \in \mathcal{Z}$ be a monomial syzygy - note that, as will be the case in the argument below, one may often have $u_{l}=v_{l^{\prime}}$, etc.

First, one can assume that no coordinate of $\mathbf{z}$ vanishes. For, otherwise, $\mathbf{z}$ would be either a (monomial) syzygy of some (connected) proper subgraph or else the sum of two such; in any case we would be done by induction on the number of edges of $G$. This assumption implies, in particular, that all vertices of $G$ have degree at least 2 and that any two edges adjacent to the same vertex belong to a cycle.

The proof will consist of various steps with loop-like alternatives.

Step 1. Due to the above form of the transposed jacobian matrix, there exists a relation $\sum_{j=1}^{d_{1}} g_{1, u_{j}} Y_{u_{j}}=0$. By looking at one such relation that effectively involves $Y_{u_{1}}$ and has smallest number of nonzero summands, one sees that there exists at least one $j \neq 1$ such that $Y_{u_{j}} \mid g_{1, u_{1}}$. Moreover, the monomials $g_{1, u_{1}} / Y_{u_{j}}$ and $g_{1, u_{j}} / Y_{u_{1}}$ share the same $\mathbf{X}$-part. On the other hand, since $Y_{u_{j}}$ is a vertex of degree at least 2, then, by the same token, there exists an entry $X_{i_{1}}$ in the $Y_{u_{1}}$-row such that $X_{i_{1}} \mid g_{1, u_{1}}$. Therefore, $X_{i_{1}} Y_{u_{j}} \mid g_{1, u_{1}}$ and $X_{i_{1}} Y_{u_{1}} \in I(G)$.

Alternative 1.1. $X_{i_{1}} Y_{u_{j}} \in I(G)$.

Then $G$ contains the 4-cycle $X_{1} Y_{u_{1}}, X_{i_{1}} Y_{u_{1}}, X_{i_{1}} Y_{u_{j}}, X_{1} Y_{u_{j}}$. Letting $F \in k[\mathbf{T}]$ denote the corresponding binomial (relation), one considers the syzygy $\mathbf{z}-f_{1, u_{1}} \lambda(F) \in$ $\mathcal{Z}$, where $g_{1, u_{1}}=f_{1, u_{1}} X_{i_{1}} Y_{u_{j}}$. Clearly, the first coordinate of this new syzygy vanishes; hence we are through by the above proviso and the inductive assumption.

Alternative 1.2. There are no 4-cycles of the form $X_{1} Y_{u_{1}}, X_{i_{1}} Y_{u_{1}}, X_{i_{1}} Y_{u_{j}}, X_{1} Y_{u_{j}}$ such that $X_{i_{1}} Y_{u_{j}}$ divides $g_{1, u_{1}}$.

Thus, let $Y_{u_{j}}$ be such that $X_{1} Y_{u_{j}} \in I(G)$ and let $X_{i_{1}}$ be such that $X_{i_{1}} Y_{u_{j}} \mid g_{1, u_{1}}$, but $X_{i_{1}} Y_{u_{j}} \notin I(G)$. We then loop back to the first step:

Step 2. Apply Step 1 to the $X_{i_{1}}$-block and $g_{i_{1}, u_{1}}$ as pivotal coordinate. Then, there exists an entry $Y_{v_{j_{1}}}$ in the $X_{i_{1}}$-row, with $v_{j_{1}} \neq u_{1}$, such that $Y_{v_{j_{1}}} \mid g_{i_{1}, u_{1}}$. Since 
$X_{i_{1}} Y_{v_{j_{1}}} \in I(G)$, then $v_{j_{1}} \neq u_{j}$ as well, by our present assumption. Since we already had $X_{i_{1}} Y_{u_{j}} \mid g_{1, u_{1}}$, we now have in fact that $X_{i_{1}} Y_{u_{j}} Y_{v_{j_{1}}} \mid g_{1, u_{1}}$.

In this situation, the claim is that there exists an entry $X_{i_{2}}$ appearing in the $Y_{u_{j}}$-row, but not in the $Y_{u_{1}}$-row, such that $X_{i_{2}} \mid g_{1, u_{1}}$. For that, one resorts to the $Y_{u_{j}}$-row and coordinate $g_{1, u_{j}}$ as pivot. Since there are no 4-cycles as just stated, then no $X$-entry in this row, other than $X_{1}$, can appear simultaneously in the $Y_{u_{1}}$-row. Therefore, there exists indeed $X_{i_{2}}$ such that $i_{2} \neq 1, i_{2} \neq i_{1}$ and such that $X_{i_{2}} \mid g_{1, u_{j}}$. On the other hand, in Step 1, as remarked before, the monomials $g_{1, u_{1}} / Y_{u_{j}}$ and $g_{1, u_{j}} / Y_{u_{1}}$ share the same $\mathbf{X}$-part. Hence, $X_{i_{2}} \mid g_{1, u_{1}}$, as claimed.

Thus, summing up, one has so far obtained that $X_{i_{1}} Y_{v_{j_{1}}} X_{i_{2}} Y_{u_{j}}$ divides $g_{1, u_{1}}$. Now it is time to loop back to Alternative 1.1, namely:

Alternative 2.1. $X_{i_{2}} Y_{v_{j_{1}}} \in I(G)$.

In this case, there is a 6 -cycle in $G$, namely, the one with edges

$$
X_{1} Y_{u_{1}}, X_{i_{1}} Y_{u_{1}}, X_{i_{1}} Y_{v_{j_{1}}}, X_{i_{2}} Y_{v_{j_{1}}}, X_{i_{2}} Y_{u_{j}}, X_{1} Y_{u_{j}} .
$$

One then considers the syzygy $\mathbf{z}-f_{1, u_{1}} \lambda(F)$, where $F \in k[\mathbf{T}]$ denotes the binomial (of degree 3 ) corresponding to the above 6-cycle and

$$
g_{1, u_{1}}=f_{1, u_{1}} X_{i_{1}} Y_{v_{j_{1}}} X_{i_{2}} Y_{u_{j}} g_{1, u_{1}} .
$$

As before, we are done by the inductive hypothesis.

Alternative 2.2. There are no 6-cycles involving the path $X_{1} Y_{u_{j}}, X_{1} Y_{u_{1}}, X_{i_{1}} Y_{u_{1}}$, $X_{i_{1}} Y_{v_{j_{1}}}$ for which an edge of the 1-factor of the cycle containing $X_{1} Y_{u_{1}}$ (other than $\left.X_{1} Y_{u_{1}}\right)$ divides the coordinate $g_{1, u_{1}}$.

We may, therefore, assume that we have a path $X_{1} Y_{u_{1}}, X_{i_{1}} Y_{u_{1}}, X_{i_{1}} Y_{v_{j_{1}}}, X_{i_{2}} Y_{u_{j}}$, $X_{1} Y_{u_{j}}$, but $X_{i_{2}} Y_{v_{j_{1}}} \notin I(G)$. We then loop back to the first step, namely:

Step 3. Apply Step 1 to the $X_{i_{2}}$-block with $g_{i_{1}, v_{j_{1}}}$ as pivotal coordinate. By a similar token, one finds new variables $X_{i_{3}}, Y_{w_{j_{2}}}$ such that $X_{i_{3}} Y_{w_{j_{2}}} X_{i_{1}} Y_{v_{j_{1}}} X_{i_{2}} Y_{u_{j}}$ divides $g_{1, u_{1}}$ (In particular, one has that if $\operatorname{deg}\left(g_{1, u_{1}}\right) \leq 5$ in Step 2, then Alternative 2.1 would necessarily take place for some $X_{i_{2}} Y_{v_{j_{1}}}$ ). Next, one searches for an 8-cycle for which the edges

$$
X_{1} Y_{u_{1}}, X_{i_{1}} Y_{v_{j_{1}}}, X_{i_{2}} Y_{w_{j_{2}}}, X_{i_{3}} Y_{u_{j}}
$$

form a 1-factor and their product divides $g_{1, u_{1}}$.

The procedure comes to a halt since, if the total degree (in $k[\mathbf{X}]$ ) of the coordinate $g_{1, u_{1}}$ is $s$, one can scan all cycles containing any (even, non-closed) path, whose order is at most $s+2$. The parenthetical observation in Step 3 just above illustrates why eventually one loops back to Step 1 .

This proves the surjectivity of the polar map $\bar{\lambda}$, showing that $\mathcal{Z}$ is generated by the polar syzygies.

One now proceeds to show that this map is injective.

For that, first note that, as a consequence of the surjectivity just proved and Lemma $2.4, \mathcal{Z}$ is generated by the polar syzygies coming from primitive cycles. Therefore we wish to show that, for each $q \geq 2$, the polar syzygies of degree $2(q-1)$ are $k$-linearly independent modulo $(\mathbf{X}, \mathbf{Y})_{2} \mathcal{Z}_{2(q-2)}$. Thus, let $\mathbf{z}=\sum_{s} \eta_{s} \mathbf{z}_{s}$, where $\mathbf{z}_{s} \in \mathcal{Z}_{2(q-1)}$ are polar syzygies coming from distinct primitive cycles (of order $2 q)$, with $\eta_{s} \in k$, be such that $\mathbf{z} \in(\mathbf{X}, \mathbf{Y})_{2} \mathcal{Z}_{2(q-2)}$. We induct on $q$. For $q=2$, we are given that $\sum_{s} \eta_{s} \mathbf{z}_{s}=0$. Since we are dealing with squares, corresponding 
coordinates of distinct $z_{s}, z_{s^{\prime}}$ are relatively prime. Therefore, no cancellation is possible; hence $\eta_{s}=0$ for every $s$.

Thus, assume that the assertion is true for polar syzygies coming from cycles of order less than $2 q$. Suppose one can write

$$
\sum_{s} \eta_{s} \mathbf{z}_{s}=\sum_{k_{1}} G_{k_{1}} \mathbf{z}_{k_{1}}+\ldots+\sum_{k_{q-2}} G_{k_{q-2}} \mathbf{z}_{k_{q-2}}
$$

where $G_{k_{t}} \in(\mathbf{X}, \mathbf{Y})_{2 t}$ and $\mathbf{z}_{k_{t}} \in \mathcal{Z}_{2(q-t-1)}$ are also polar syzygies coming from primitive cycles, for $1 \leq t \leq q-3$.

Now, a primitive cycle is determined by any one of its 1-factors. Moreover, any nonzero $\left(i, i^{\prime}\right)$-coordinate of a polar syzygy corresponding to a cycle $C$ of $G$ is obtained by multiplying out all the "edges", other than the $\left(i, i^{\prime}\right)$ th edge, of the unique 1-factor of $C$ containing the $\left(i, i^{\prime}\right)$ th edge. It follows that distinct cycles $\mathbf{z}_{s}, \mathbf{z}_{s^{\prime}}$ have distinct monomial parts on corresponding coordinates. Therefore, no cancellation of corresponding nonzero coordinates is possible on the left-hand side of (4); hence any nonzero coordinate on the left-hand side, say, $\eta_{1}$, has to be cancelled against some nonzero coordinate of the same name on the right-hand side of (4). The latter is of the form $G_{k_{t}} F_{k_{t}}$, where $F_{k_{t}}$ is the product of all edges (but one) of the 1-factor of a smaller primitive cycle. Since we are looking at corresponding coordinates, this is impossible unless the smaller cycle is obtained from $\mathbf{z}_{1}$ by means of a chord of the cycle corresponding to $\mathbf{z}_{1}$. This gives a contradiction, since the latter is chordless by hypothesis.

2.2. Second Betti number and complete intersections. Given a connected graph $G$, let

$$
0 \rightarrow R^{b_{r}} \rightarrow \cdots \rightarrow R^{b_{2}} \rightarrow R^{b_{1}} \rightarrow R^{b_{0}} \rightarrow \mathfrak{J}(G) \rightarrow 0
$$

be a minimal free resolution of its jacobian module over the polynomial ring $R=$ $k[\mathbf{X}]$. The number $b_{j}=b_{j}(\mathfrak{J}(G))$ is known as the $j$ th Betti number of the module $\mathfrak{J}(G)$. Clearly, $b_{0}=n$ and $b_{1}$ are the vertex number and the edge number of $G$, respectively.

The number of primitive cycles of $G$ will be denoted by $f \operatorname{rank}(G)$. Note that $f \operatorname{rank}(G) \geq \operatorname{rank}(G)$, where $\operatorname{rank}(G)$ stands for the usual graph theoretic rank of $G$ (cf. [1]).

These numbers have a natural meaning in terms of the homology of $\mathfrak{J}(G)$ if $G$ is bipartite. Let $k[G] \subset R=k[\mathbf{X}]$ denote the $k$-subalgebra generated by the generators of the edge-ideal $I(G)$. As previously, let $J \subset k[\mathbf{T}]$ denote the ideal of polynomial relations of $k[G]$.

The main result is the following.

Theorem 2.5. Let $G$ be a connected bipartite graph and let

$$
0 \rightarrow R^{b_{r}} \stackrel{\Theta_{r}}{\rightarrow} \cdots \rightarrow R^{b_{2}} \stackrel{\Theta_{2}}{\rightarrow} R^{b_{1}} \stackrel{\Theta_{1}}{\rightarrow} R^{n} \rightarrow \mathfrak{J}(G) \rightarrow 0
$$

be a minimal free resolution of its jacobian module over the polynomial ring $R=$ $k[\mathbf{X}]$, with $\Theta_{1}={ }^{t} \Theta(G)$. Then:

(i) $b_{2}=f \operatorname{rank}(G)$.

(ii) The following conditions are equivalent:

(a) $\mathfrak{J}(G)$ has homological dimension at most 2

(b) $f \operatorname{rank}(G)=\operatorname{rank}(G)$

(c) $k[G]$ is a complete intersection. 
Proof. (i) By Theorem 2.3, one has $\mu(J)=\mu(\mathcal{Z})$, and by Lemma 2.4, $\mu(J)=$ $f \operatorname{rank}(G)$. Therefore, $b_{2}(=\mu(\mathcal{Z}))=f \operatorname{rank}(G)$, as required.

(ii) (a) $\Rightarrow$ (b) If $\mathfrak{J}(G)$ has homological dimension at most 2 then, by Schanuel's lemma applied to the above resolution, $\operatorname{ker} \Theta_{1}$ is a free homogeneous submodule of $R^{b_{1}}$ of $\operatorname{rank} b_{1}-\operatorname{rank}\left(\Theta_{1}\right)=b_{1}-n+1=\operatorname{rank}(G)$ by Theorem 1.3 and [1]. By part (i), it follows that $b_{2}=\operatorname{rank}(G)$.

(b) $\Rightarrow$ (c) One has $f \operatorname{rank}(G)=b_{2}$ by (i). Therefore, it suffices to show that $\operatorname{rank}\left(\Theta_{2}\right)=$ ht $J$ (the codimension of the ideal $J$ of polynomial relations of $k[G]$ ). But since $G$ is bipartite, one has $\operatorname{dim} k[G]=\operatorname{rank} \Phi(G)=n-1$. Therefore, $n-1=$ $\# \mathbf{T}-$ ht $J=b_{1}-$ ht $J$; hence ht $J=b_{1}-n+1=\operatorname{rank}\left(\Theta_{2}\right)$.

(c) $\Rightarrow$ (a) The assumption means that $\mu(J)=\operatorname{ht}(J)$. From part (i) (and its proof), one knows that $b_{2}=\mu(J)$ and $\operatorname{rank}\left(\Theta_{2}\right)=$ ht $J$. It follows that $b_{2}=$ $\operatorname{rank}\left(\Theta_{2}\right)$; hence $\Theta_{2}$ yields an injective map.

\section{REFERENCES}

1. F. Harary, Graph Theory, Addison-Wesley Publishing Co., Reading, Mass., 1969. MR 41:1566

2. L. R. Doering and T. Gunston, Algebras arising from bipartite planar graphs, Comm. Algebra 24 (1996), 3589-3598. CMP 96:17

3. A. Simis, W. V. Vasconcelos and R. Villarreal, On the ideal theory of graphs, J. Algebra 167(2) (1994), 389-416. MR 95e:13002

4. R. Villarreal, Cohen-Macaulay graphs, Manuscripta Math. 66 (1990), 277-293. MR 91b:13031

5. R. Villarreal, Rees algebras of edge-ideals, Comm. Algebra 23 (1995), 3513-3524. MR 96e: 13005

Instituto de Matemática, Universidade Federal da Bahia, Av. Ademar de Barros, S/N, 40170-210 SAlvador, BA, Brazil

E-mail address: aron@ufba.br 